\title{
Inconsistent conclusions on QoL outcomes from the same clinical trial
}

\author{
Stefan Lange $^{1} \cdot$ Jan Gaertner $^{2} \cdot$ Natalie McGauran $^{1} \cdot$ Nobert Schmacke $^{3}$. \\ Vera Weingärtner ${ }^{1,4}$
}

Received: 24 December 2014 / Accepted: 25 February 2015/Published online: 18 April 2015

(c) The International Gastric Cancer Association and The Japanese Gastric Cancer Association 2015

Letter to the editor on the article by Kripp et al. "Quality of life of older adult patients receiving docetaxel-based chemotherapy triplets for esophagogastric adenocarcinoma: a randomized study of the Arbeitsgemeinschaft Internistische Onkologie (AIO)," Gastric Cancer (2014) 17:181-187.

There is a lack of quality-of-life (QoL) data from cancer trials [1,2]. Therefore, the article by Kripp et al. [3] is to be welcomed. However, we have some queries and would like to see the following issues addressed by the authors:

1. Kripp et al. [3] present QoL findings from the FL0T $65+$ study. Results from this study have previously been presented by Al-Batran et al. [4]. The study evaluated triple- versus double-drug chemotherapy in elderly patients with esophagogastric cancer [FLOT (including docetacel) vs. FLO]. According to the methods section in the Al-Batran et al. study, "The primary objective of the study was tolerability and feasibility, defined as per group differences in toxic effects (etc.) and the proportions of patients with $\geq 10$ point change of QoL global health status at 8 weeks compared to baseline." According to the results, “... a moderate to large ( $\geq 10$ points) deterioration of $\mathrm{QoL}$ global health status scores during the first 8 weeks of

Stefan Lange

stefan.lange@iqwig.de

1 Institute for Quality and Efficiency in Health Care (IQWiG), Im Mediapark 8, 50670 Cologne, Germany

2 Department of Palliative Care, University Medical Center Freiburg, Freiburg, Germany

3 Institute for Public Health and Nursing Research (IPP), University of Bremen, Bremen, Germany

4 Department of Palliative Medicine, University Hospital of Cologne, Cologne, Germany treatment occurred in 19 of $40(47.5 \%)$ evaluable patients with FLOT compared to 9 of 44 (20.5\%) evaluable patients with FLO $(p=.011)$." Al-Batran et al. conclude that "...QQL deteriorated in a relevant proportion of patients."

2. In contrast, in Kripp et al., "the primary endpoint of the QoL assessment was time to definitive deterioration from baseline by $5 \%$ on the GHS/QOL scale." Various secondary QoL endpoints are also defined. The difference between treatment groups is no longer significant: "Particularly, there was no significant difference regarding time to definitive deterioration of global health status/quality of life from baseline (primary endpoint)." Kripp et al. reach a rather different conclusion on QoL: “...no negative impact of the addition of docetaxel on QOL parameters could be demonstrated." Kripp et al. refer to Al-Batran et al. with regard to study design and report the result for the statistically significant difference at week 8 (and also refer to this result in the discussion). However, they mention neither that the data shown refer to the endpoint defined as the main QoL endpoint in [4] nor the conflicting conclusion. The registry entry for FLOT $65+$ in ClinicalTrials.gov provides no answer to the question of the main QoL endpoint as no operationalization of QoL is available [5].

3. No funding statement is included by Kripp et al. In contrast, Al-Batran et al. state that "The study was partially funded by Sanofi-Aventis." The main author (who is a co-author in Kripp et al.) discloses a potential conflict of interest, which is not disclosed in Kripp et al.

We kindly ask Kripp et al. to explain the above inconsistencies. Furthermore, we suggest that Kripp et al. make 
clear what exactly was written in the original study protocol of FLOT $65+$ and also disclose the full protocol.

Clinical studies should be reported in accordance with the study protocol. We call for the regular publication of full protocols to help identify inaccurate and selective reporting.

\section{References}

1. Basch E. Toward patient-centered drug development in oncology. N Engl J Med. 2013;369(5):397-400.

2. Amdal CD, Jacobsen AB, Guren MG, Bjordal K. Patient-reported outcomes evaluating palliative radiotherapy and chemotherapy in patients with oesophageal cancer: a systematic review. Acta Oncol. 2013;52(4):679-90.

3. Kripp M, Al-Batran SE, Rosowski J, Pauligk C, Homann N, Hartmann JT, et al. Quality of life of older adult patients receiving docetaxel-based chemotherapy triplets for esophagogastric adenocarcinoma: a randomized study of the Arbeitsgemeinschaft Internistische Onkologie (AIO). Gastric Cancer. 2014;17(1):181-7.

4. Al-Batran SE, Pauligk C, Homann N, Hartmann JT, Moehler M, Probst S, et al. The feasibility of triple-drug chemotherapy combination in older adult patients with oesophagogastric cancer: a randomised trial of the Arbeitsgemeinschaft Internistische Onkologie (FLOT65 +). Eur J Cancer. 2013;49(4):835-42.

5. ClinicalTrials.gov. Oxaliplatin and 5-fluorouracil with or without docetaxel in elderly patients ( $>65$ years) with stomach and esophagus cancer. ClinicalTrials.gov Identifier: NCT00737373 (online). 2012. http://clinicaltrials.gov/ct2/show/NCT00737373? term=NCT00737373\&rank=1. Accessed 11 Dec 2014. 